\title{
Nickel and Strontium Nitrates as Modifier for the Determination of Selenium in Urine by Zeeman Platform Graphite-Furnace Atomic Absorption Spectrometry
}

\author{
Yan-Zhong Liang*十, Mei LI** and Zhu RAo** \\ ${ }^{*}$ Research Center for Eco-Environmental Sciences, Academia Sinica, P. O. Box 2871, \\ Beijing, P. R. China, 100085 \\ **National Research Center for Geoanalysis, Beijing, P. R. China, 100037
}

\begin{abstract}
A mixture of nickel and strontium nitrates has been proved to be suitable and feasible for the determination of selenium in urine by graphite-furnace atomic absorption spectrometry with respect to the thermal stabilization and minimization of urine matrix interferences. Thermal pretreatment temperature of $1400^{\circ} \mathrm{C}$ and $900^{\circ} \mathrm{C}$ can be utilized in aqueous standard solution and 1:3 diluted urine with the proposed modifier, respectively. The results demonstrate that nickel and strontium nitrate mixed modifier can significantly remove or depress troublesome interference caused by sulfate, phosphate and concomitant metal ions. The graphite furnace temperature program and modifier mass have been optimized. The amount of mixed modifier required for stabilizing selenium and suppressing the effect of urine matrix is markedly lower than those documented in the literature, which reduces the risk of graphite furnace contamination. The effects of different atomizer surfaces on the selenium sensitivity were compared in this work. Better recoveries were obtained for different urine samples in the presence of nickel and strontium nitrates.
\end{abstract}

Keywords Selenium, nickel, strontium, chemical modifier, urine, graphite-furnace atomic absorption spectrometry

According to the Environmental Health Criteria 58 of the World Health Organization ${ }^{1}$, chronic dietary lack of selenium, as a common phenomenon, has been associated with various degenerative diseases despite the fact that a symptomatic deficiency of selenium in human is rare. Biological markers of selenium nutritional status ${ }^{2}$ include the selenium concentration in whole blood, plasma and urine, which reflect the recent intake. Due to the environmental and biological significance of selenium, much interest has been focused on the determination of this element ${ }^{3-5}$ as well as studies of atomization and interference mechanism in recent years. $^{6-9}$ The very low detection limit that can be achieved with graphite-furnace atomic absorption spectrometry (GFAAS) has made this technique one of the most widely applied methods to a Se measurement. However, certain shortcomings in the analysis of a volatile element like selenium are the loss of analyte by volatilization during thermal pretreatment at temperatures as low as $200^{\circ} \mathrm{C}^{10}$, and troublesome interferences caused by sample constitutes, which could yield a background that in some cases can not be corrected, even when a Zeeman effect-based apparatus is employed. ${ }^{3}$

$\dagger$ To whom correspondence should be addressed. Present address: Department of Geological Sciences, The University of Illinois at Chicago, $845 \mathrm{~W}$ Taylor St., IL 60607, USA.
The introduction of stabilized temperature platform furnace (STPF) concept, first suggested by Slavin et al. ${ }^{11}$, which allows atomization under near-isothermal conditions, has alleviated these drawbacks to some extent. As a part of the STPF conditions, the use of chemical modifiers has been proved to be indispensable for stabilizing volatile selenium in the pyrolysis step. To date, numerous chemical modifiers have been reported, including a single metal, such as nickel ${ }^{12}$, palladium ${ }^{13}$, copper ${ }^{14}$, silver ${ }^{15}$ or molybdenum ${ }^{16}$, as well as a mixture such as palladium plus magnesium ${ }^{17}$, palladium plus strontium $^{6}$, or nickel plus copper. ${ }^{10}$ In spite of studies concerning the thermal stabilization of selenium and its different chemical species, number of reports involving an interference-free determination of selenium has been recognized to be particularly difficult to determine in urine $^{\mathrm{Il}}$ due to its higher volatility and susceptibility to interference ions.

The aim of this work was to study the effect of nickel and strontium as thermal stabilizer in a graphite furnace on the selenium determination in urine under STPF conditions with a Zeeman background correction, as well as its effectiveness in a furnace reduction of iron, sulfate, phosphate and other potential interferences. 


\section{Experimental}

\section{Equipment}

A Hitachi Z-8200 Zeeman atomic absorption spectrophotometer equipped with a GA-3 graphite furnace and an SSC-300 auto-sampler was used for all atomic absorption measurements. Acquisition and treatment of data were performed with an IBM compatible 80386DX computer and Z-8200 windows-based application software, data and absorbance profiles were output through a Brother M-2724 printer. The wavelength, spectral bandwidth and the lamp current for selenium determination were based on the recommendation of instrument manufacturer. Pyrolytic graphite coated graphite tubes with a pyrolytic graphite platform was employed throughout the experiment. When chemical modifiers were used, it was added as a separate solution by use of the automatic addition feature of the SSC- 300 .

Sample and modifier aliquots were $10 \mu \mathrm{l}$ in all cases. The measurement of absorbance signals was carried out based on the mode of peak area, and internal gas flow was interrupted during atomization stage. A typical graphite furnace temperature program for the determination of selenium in urine is given in Table 1.

\section{Reagents}

All reagents were of the highest available purity but at least of analytical-reagent grade.

A selenium stock solution of $1 \mathrm{mg} / \mathrm{ml}$ was prepared by dissolving $0.1405 \mathrm{~g} \mathrm{SeO}_{2}$ (Analytical reagent grade, Beijing Chemical Ltd., P. R. China) in $20 \mathrm{ml}$ of $4 \mathrm{M} \mathrm{HCl}$ and diluting to $100 \mathrm{ml}$ with deionized water. Working standard solutions were obtained by further dilution to volume with deionized water. Stock solution of nickel $(10 \mathrm{mg} / \mathrm{ml})$ was prepared from analytical reagent grade nickel nitrate (Beijing Chemical Ltd., P. R. China). A strontium nitrate solution was prepared by dissolving strontium carbonate (Specpure, Johnson Mathey Chemical Ltd.) in dilute nitric acid.

\section{Procedure}

Fresh urine samples used in this experiment were collected from local volunteers, and were further diluted to $1: 3$ with deionized water prior to measurement. The samples were stored in polyethylene bottles and refrigerated at $4^{\circ} \mathrm{C}$.

\section{Results and Discussion}

Based on a previous study ${ }^{18}$, nickel has an enhancement effect on Se. We thus check the possibility of $\mathrm{Ni}$ plus $\mathrm{Sr}$ as a chemical modifier for Se determination in this study.

Effectiveness of Ni+Sr as a chemical modifier for the determination of Se in urine

The thermal pretreatment and atomization curves for
Table 1 Graphite-furnace temperature program for the determination of $\mathrm{Se}$ in urine using a nickel+strontium modifier

\begin{tabular}{lrrrrr}
\hline Parameter & 1 & 2 & 3 & 4 & 5 \\
Temperature $/{ }^{\circ} \mathrm{C}$ & 130 & 1300 & 2200 & 2700 & 30 \\
Ramp time $/ \mathrm{s}$ & 10 & 10 & 0 & 1 & 1 \\
Hold time $/ \mathrm{s}$ & 30 & 80 & 5 & 5 & 5 \\
Gas flow $/ \mathrm{ml} \mathrm{min}$ mi $^{-1}$ & 200 & 200 & 0 & 200 & 200 \\
\hline
\end{tabular}

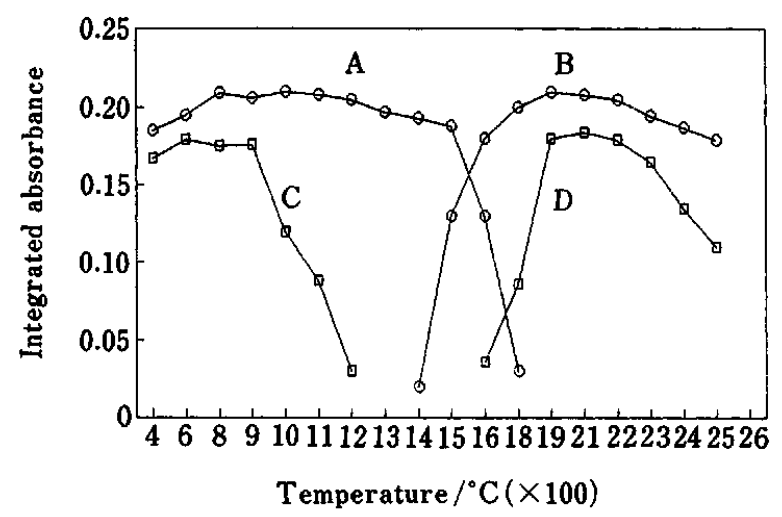

Fig. 1 Ashing (curves $A$ and $C$ ) and atomization (curves $B$ and D) curves of $2 \mathrm{ng} \mathrm{Se}$ in water (curves $\mathrm{A}$ and $\mathrm{B}$ ) and $1: 3$ diluted urine (curves $\mathrm{C}$ and $\mathrm{D}$ ) with $\mathrm{Ni}$ plus $\mathrm{Sr}$ as a chemical modifier.

selenium in this work with the proposed nickel and strontium nitrates mixed modifier in both water and urine are depicted in Fig. 1 all data have been blank corrected. From these curves in Fig. 1, it appears to be obvious that the proposed modifier has a substantial stabilization effect on selenium in an aqueous standard and urine. The maximum applicable pyrolysis temperature for a loss-free thermal pretreatment may be up to $1400^{\circ} \mathrm{C}$ in water (curve A). By contrast, the loss of selenium begins to occur in urine matrix (curve $\mathrm{C}$ ) when the charring temperature exceeds $900^{\circ} \mathrm{C}$, which is in agreement with Carnick et al. ${ }^{4}$ Deionized water and four-fold diluted urine were spiked with $2 \mathrm{ng}$ of selenium. It is thus clear that the difference in sensitivity for selenium in water and in diluted urine results from losses from the urine during the pyrolysis step. Besides stabilization to higher thermal pretreatment temperature, the application of a modifier usually permits the use of higher atomization temperatures. ${ }^{17}$ The influence of nickel and strontium nitrates mixed modifier on atomization temperature for a selenium determination seems to be uniformly in one direction, as can be seen in curve $B$ and $D$ in Fig. 1; namely, the integrated absorbances tend to decrease when atomization temperatures exceed $2300^{\circ} \mathrm{C}$, and the lowest applicable temperature for atomization is $1900^{\circ} \mathrm{C}$. The timeresolved absorbance profiles for selenium, as shown in Fig. 2, indicate that the selenium absorbance in the urine 


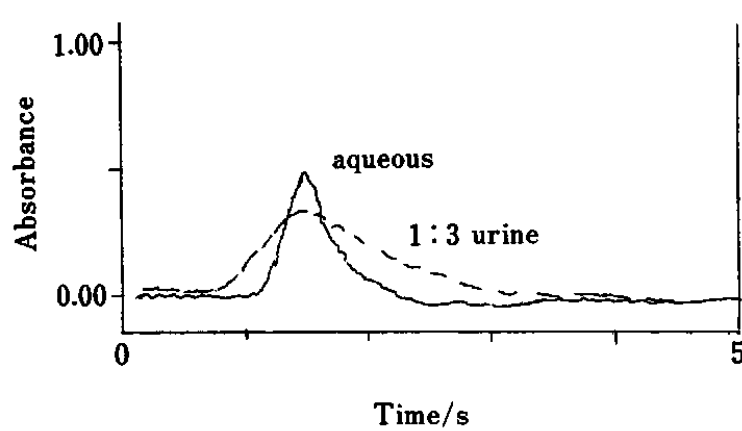

Fig. 2 Absorbance profiles for $2 \mathrm{ng}$ Se in an aqueous solution (solid line), and $1: 3$ urine (broken line) with $\mathrm{Ni}-\mathrm{Sr}$ modifier. The pyrolysis is at $900^{\circ} \mathrm{C}$, and atomization is at $2100^{\circ} \mathrm{C}$.

starts to appear in the atomization stage some $0.2 \mathrm{~s}$ earlier than that in water at a pyrolysis temperature of $900^{\circ} \mathrm{C}$, and atomization temperature of $2100^{\circ} \mathrm{C}$, which is in conformity with the literature. ${ }^{4}$ However, an apparent discrepancy between the absorbance profiles in Fig. 2 and those reported in ref. 4 is that the absorbance profile of selenium in urine becomes flat as compared with that in an aqueous standard. This could be ascribed to the fact that the four-fold diluted urine samples used in this work contained higher contents of matrix components, as reported in ref. 4. It should be noticed that the profiles of the atomization pulses do not necessarily possess the optimum shape at the optimum atomization temperatures at which the highest integrated absorbance is obtained. In this report, the graphite-furnace temperature program was basically optimized according to the criterion of maximum integrated absorbance.

The stabilization mechanism of selenium by nickel and strontium mixture can possibly be ascribed to the formation of NiSe according to Ediger ${ }^{12}$ and Styris. ${ }^{19}$

\section{Optimization of the mass of the modifier}

It has well been recognized that the modifier mass has a pronounced bilateral influence on the determination of volatile elements. On the one hand, a higher thermal pretreatment temperature can be achieved with a higher modifier mass; ${ }^{20}$ on the other hand, higher mass of a modifier generally decreases the signal due to secondary adsorption at the cooler ends of the graphite tube. ${ }^{21,22}$ Hence, a compromise choice of the modifier mass was deemed to be essential in order to achieve the maximum of sensitivity and thermal stabilization. The influence of increasing mass of nickel and strontium nitrates on the selenium signals is shown in Fig. 3 , in which it need to be mentioned that the mass of the modifier is referred to as the separate mass of nickel and strontium nitrate, not the sum of the both. It appears to be true from Fig. 3 that the effect of the mass of the modifier on the integrated absorbance of selenium is different in both aqueous solution and a urine matrix as well as the discrepancy in the sensitivity. In the case of a water matrix, a maximum of the integrated absorbance for selenium can

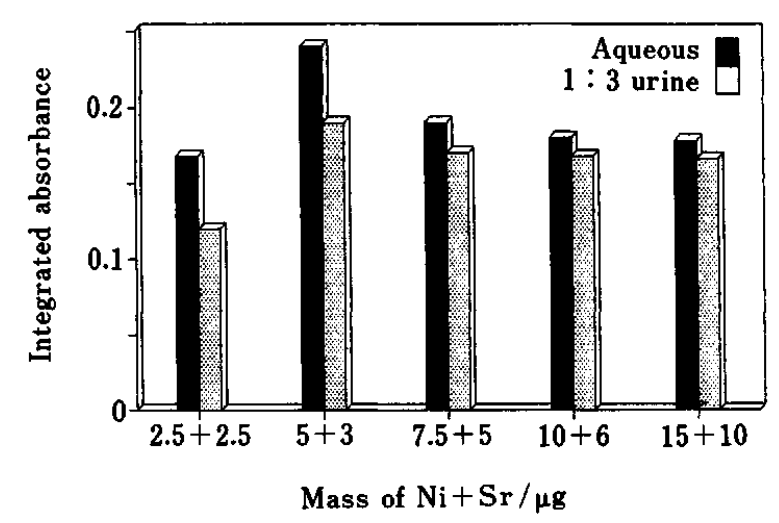

Fig. 3 Effect of the mass of the modifier on the integrated absorbance of $2 \mathrm{ng}$ of $\mathrm{Se}$ in water and urine. Atomization at $2200^{\circ} \mathrm{C}$, and pyrolysis at $800^{\circ} \mathrm{C}$.

be observed along with the increase in the mass of the modifier. Meanwhile, when the masses of nickel plus strontium exceed 7.5 plus $5 \mu \mathrm{g}$, the absorbance demonstrates a slight decrease. In the case of a urine matrix, two distinguishable features can be noted in comparison with the former case from Fig. 3, despite the fact that the overall tendency of the effect is similar. First, the absorbance signal of selenium has probably been reduced by a urine matrix when nickel of $2.5 \mu \mathrm{g}$ and $2.5 \mu \mathrm{g}$ strontium modifier were injected onto a platform. Second, the integrated absorbance of selenium tends to remain stable when the masses of nickel and strontium exceed $5 \mu \mathrm{g}$ and $3 \mu \mathrm{g}$, respectively. Nickel of $5 \mu \mathrm{g}$ and strontium of $3 \mu \mathrm{g}$ is likely to be sufficient to stabilize selenium in $1: 3$ diluted urine. The mass of the modifier is greatly lower than those recommended in literature ${ }^{4,8}$, which reduces the possibility of nickel contamination on the graphite furnace.

\section{Interference studies}

The difficulty in measuring selenium in urine can be correlated with its volatility, interaction with graphite furnace, and spectral and chemical interferences. ${ }^{23}$ The influence of some potential interference ions in urine on the signal of the analyte element was studied in this experiment. The relative integrated absorbances obtained in comparison with the interference-free solution and the relative standard deviations calculated for five replicate determinations are listed in Table 2.

The charring loss of selenium caused by sulfate has been proven to be particularly troublesome ${ }^{6}$, and workers have reported various permissible amounts. Welz et al. ${ }^{9}$ found that sulfate of $15 \mu \mathrm{g}$ in the form of sodium sulfate would give rise to $5 \%$ depression on selenium signal with a palladium nitrate-magnesium nitrate-barium nitrate modifier. $\mathrm{Ni}$ et al..$^{6}$ in recent paper recommended palladium plus strontium for the minimization of sulfate to be up to $20 \mu \mathrm{g}$. In this work, the maximum of tolerable sulfate was $25 \mu \mathrm{g}$ with nickel and strontium nitrate mixed modifier, which only causes 
Table 2 Interference studies for the determination of Se in urine

\begin{tabular}{llcc}
\hline Interferent & $\begin{array}{c}\text { Compound } \\
\text { used }\end{array}$ & $\begin{array}{c}\text { Interferent } \\
\text { concentration/ } \\
\text { mg ml }\end{array}$ & Recovery, \% \\
\hline $\mathrm{Fe}^{3+}$ & $\mathrm{FeCl}_{3}$ & 3.0 & $97 \pm 3$ \\
$\mathrm{SO}_{4}{ }^{2-}$ & $\mathrm{K}_{2} \mathrm{SO}_{4}$ & 2.5 & $85 \pm 5$ \\
$\mathrm{PO}_{4}{ }^{3-}$ & $\mathrm{Na}_{3} \mathrm{PO}_{4}$ & 0.6 & $102 \pm 5$ \\
$\mathrm{Ca}^{2+}$ & $\mathrm{Ca}\left(\mathrm{NO}_{3}\right)_{2}$ & 0.75 & $98 \pm 6$ \\
$\mathrm{ClO}_{4}{ }^{-}$ & $\mathrm{KClO}_{4}$ & 10 & $95 \pm 7$ \\
$\mathrm{Glucose}$ & & 0.5 & $84 \pm 8$ \\
\hline
\end{tabular}

All of the results are the \% integrated absorbance with the absorbance of the matrix-free reference solution being $100 \%$. ( \pm RSD for five replicate determinations). An aliquot of $10 \mu \mathrm{l} \mathrm{sample}$ was injected on the platform.

a $5-15 \%$ depression to the signal generated by $2 \mathrm{ng}$ of selenium. This data combined with previous report ${ }^{6}$ plausibly implies that strontium could become a valid modifier for eliminating the sulfate interference for selenium determination.

The high concentration of phosphates in human urine results in a spectral interference at $196.0 \mathrm{~nm}$, owing to the hyperfine structure of the molecular bands of polyatomic species, mainly $\mathrm{P}_{2}, \mathrm{PO}$ and $\mathrm{PO}_{2}$, produced by the thermal decomposition of phosphate. ${ }^{24}$ However, nickel can react with phosphate and lead to the formation of atomic phosphorus, thus diminishing the background absorption. No spectral interference of phosphate was observed in the experiment though the amount of phosphate surpassed the highest tolerable limit of $6 \mu \mathrm{g}$.

Perchlorate is a typical concomitant because organic samples are often digested and oxidized in a mixture of nitric and perchloric acids. In order to verify the extensive applicability of the proposed modifier, an attempt was made to determine the level of perchlorate that could be tolerated. The recovery $\%$ of selenium in Table 2 shows that perchlorate of $100 \mu \mathrm{g}$ has little interference in the presence of nickel and strontium.

Metals like $\mathrm{Fe}^{3+}$ and $\mathrm{Ca}^{2+}$, which are potential spectral interferents for the selenium signal did not demonstrate pronounced interference effect. The allowable amount of $\mathrm{Fe}^{3+}$ and $\mathrm{Ca}^{2+}$ with nickel modifier, as listed in Table 2, is generally sufficient for the determination of selenium in urine. Sugar as a possible interfering component ${ }^{17}$ in urine has also been tested. The recovery data indicate that $5 \mu \mathrm{g}$ sugar yields about a $15 \%$ depression on the $\mathrm{Se}$ integrated absorbance.

\section{Calibration curve and standard addition method}

The calibration curve for selenium standards prepared in $0.01 \mathrm{M}$ nitric acid and the matrix modification mixture was found to be linear from 0 to $80 \mu \mathrm{g} / 1$ with an aliquot of $10 \mu \mathrm{l}$. Figure 4 shows a representative calibration curve as well as results for standard additions to three urine samples. Due to the complicated matrix in urine and the large variation in the matrix composition among

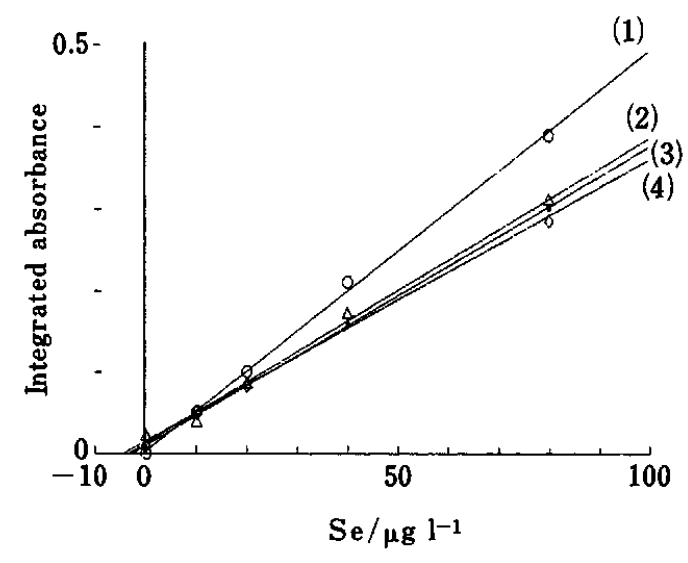

Fig. 4 Calibration curve (1) and standard addition curves (2) - (4).

the samples, the addition of a chemical modifier does not completely remove all interferences. As can be seen in Fig. 4, there was typically an approximate $10-15 \%$ difference in the slopes of the calibration curve and the standard additions to make a proper determination of selenium in urine. The comparison of standard addition curves in Fig. 4 implies that the selenium concentrations in urine samples are in the typical range of $2-8 \%$

\section{Effect of different atomizer surfaces on Se sensitivity}

Many conflicts in the literature concerning the determination of selenium deal with the properties of the furnace tubes. ${ }^{4}$ Vickrey et al. ${ }^{25}$ reported considerable influence on the selenium absorbance using different graphite surfaces, and found ${ }^{26}$ that pyrolytically coated tubes were advantageous for selenium. The results obtained by Fernandez and Lannarone ${ }^{27}$ also support the above viewpoint. However, Matousek ${ }^{28}$ stated in a review that selenium showed poorer sensitivity on pyrolytically coated tubes than uncoated tubes.

In our work, experiments were carried out to achieve further insight into this question. The bar graphs constructed with aqueous solutions are shown in Fig. 5. Clearly, no significant difference can be observed for the integrated absorbance of selenium from both wall and platform atomization, regardless of using either pyrolytic or non-pyrolytic graphite tubes. Nevertheless, the integrated absorbance of selenium atomized from the platform was drastically higher than those from the tube wall (see Fig. 5).

\section{Analytical recovery}

The recovery data of selenium added to urine with different amounts are presented in Table 3. The recovery results in urine samples from volunteers were between $86-101 \%$.

\section{Sensitivity and detection limit}

The sensitivity, expressed as characteristic mass, was $30 \mathrm{pg}$, which is in agreement with the published 


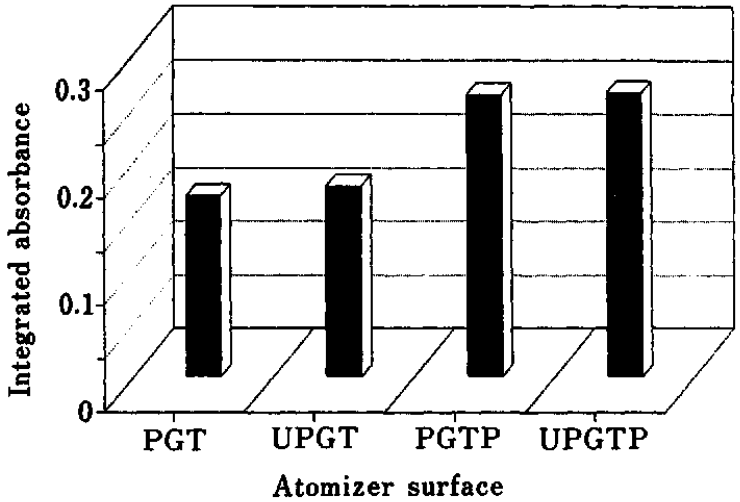

Fig. 5 Effect of the atomizer surfaces on the Se signals in water using Ni plus $\mathrm{Sr}$ modifier. Atomization at $2200^{\circ} \mathrm{C}$, and pyrolysis at $1200^{\circ} \mathrm{C}$.

Table 3 Analytical recovery of selenium spiked in urine (RSDs for 5 replicate determinations were below 10\%)

\begin{tabular}{cccc}
\hline Sample & Se Added $/ \mathrm{ng}$ & Se Found $/ \mathrm{ng}$ & Recovery, \% \\
\hline 1 & 0.5 & 0.47 & 94 \\
& 1.0 & 0.86 & 86 \\
2 & 2.0 & 1.73 & 87 \\
& 0.5 & 0.44 & 88 \\
& 1.0 & 0.90 & 90 \\
3 & 2.0 & 1.83 & 92 \\
& 0.5 & 0.505 & 101 \\
& 1.0 & 0.97 & 97 \\
\hline
\end{tabular}

values. $4,8,9$ The detection limit expressed as three-times the standard deviation of blank solution, was $6 \mu \mathrm{g} / 1 \mathrm{using}$ $10 \mu$ of the sample solution. Ten replicate injections of a urine sample spiked with $2 \mathrm{ng} \mathrm{Se}$ gave a relative standard deviation of about $5 \%$.

According to the results obtained in this experiment, it can be concluded that nickel plus strontium is a valid and feasible chemical modifier for the determination of selenium in urine by Zeeman GFAAS. The advantage of nickel plus strontium are its permitting higher pyrolysis temperatures than most common chemical modifiers such as $\mathbf{P d}$ or $\mathbf{P d}+\mathbf{M g}$, and its ability to reduce interference. The recovery data of different urine samples proves the effectiveness and applicability of the approach for the determination of selenium in urine.

This work is supported by Chinese Academy of Sciences and Beijing Hitachi Scientific Instruments Laboratory.

\section{References}

1. Selenium Environmental Health Criteria 58, World Health Organisation, Geneva, 1987.

2. J. Neve and P. Hochstein, Biochemistry, 2, 1420 (1963).

3. M. T. Petez-Corona, M. B. De la Calle-Guntinas, Y. Madrid and C. Camara, J. Anal. At. Spectrom., 10, 321 (1995).

4. G. R. Carnrick, D. C. Manning and W. Slavin, Analyst [London], 108, 1297 (1983).

5. I. Harrison and D. Littejoin, J. Anal. At. Spectrom., 10, 215 (1995).

6. Z. M. Ni, B. He and H. B. Han, Specrochim. Acta, 49B, 947 (1994).

7. A. Cedergren, I. Lindberg, E. Lundberg, D. C. Baxter and W. Frech, Anal. Chim. Acta, 180, 373 (1986).

8. F. Laborda, J. Vinuales, J. M. Mir and J. R. Castillo, J. Anal. At. Spectrom., 8, 737 (1993).

9. B. Welz, G. Bozsai, M. Sperling and B. Radzuik, J. Anal. At. Spectrom., 7, 505 (1992).

10. T. M. Mahmood, H. C. Qiao and K. W. Jackson, J. Anal. At. Spectrom., 10, 43 (1995).

11. W. Slavin, D. C. Manning and G. R. Camrick, At. Spectrosc., 2, 137 (1981).

12. R. D. Ediger, At. Absorption Newsl., 14, 127 (1975).

13. Z. M. Ni and X. Q. Shan, Spectrochim. Acta, 42B, 937 (1988).

14. F. J. Szydlowsky, At. Absorption Newsl., 16, 60 (1977).

15. J. Alexander, K. Saeed and Y. Thomssen, Anal. Chim. Acta, 120, 377 (1980).

16. E. L. Henn, Anal. Chem., 47, 428 (1975).

17. G. Schlemmer and B. Welz, Spectrochim. Acta, 41B, 1157 (1986).

18. Y. Z. Liang, M. Li and Z. Rao, Fresenius'J. Anal. Chem. (in press).

19. D. L. Styris, Fresenius'J. Anal. Chem., 323, 710 (1986).

20. P. B. Mandjukov, E. T. Vassileva and V. D. Simeonov, Anal. Chem., 64, 2596 (1992).

21. W. Frech, K. Li, M. Berglung and D. C. Baxter, J. Anal. At. Spectrom., 7, 141 (1992).

22. N. S. Thomaidis, E. A. Piperaki and C. E. Efstathiou, J. Anal. At. Spectrom., 10, 221 (1995).

23. K. S. Subramanian, Prog. Anal. At. Spectrosc., 11, 511 (1988).

24. K. Saeed and Y. Thomssen, Anal. Chim. Acta, 130, 281 (1981).

25. T. M. Vickrey, G. V. Harrison and G. R. Ramelow, Anal. Chem., 120, 1573 (1981).

26. T. M. Vickrey and M. S. Buren, Anal. Lett., 13, 1465 (1980).

27. F. J. Femandez and J. Lannarone, At. Absorption Newsl., 17, 117 (1978).

28. J. P. Matousek, Prog. Anal. At. Spectrosc., 4, 247 (1981). 